\title{
Optimization of flow diverters for cerebral aneurysms
}

\author{
Hitomi Anzai ${ }^{\mathrm{a}}$, Makoto Ohta ${ }^{\mathrm{a}}$, Jean-Luc Falcone ${ }^{\mathrm{b}}$, Bastien Chopard ${ }^{\mathrm{b}, *}$ \\ a Tohoku University, Japan \\ ${ }^{\mathrm{b}}$ University of Geneva, Switzerland
}

\section{A R T I C L E I N F O}

\section{Article history:}

Received 16 August 2011

Received in revised form

28 December 2011

Accepted 28 December 2011

Available online 13 January 2012

Keywords:

Flow diverter

Lattice Boltzmann simulations

Cerebral aneurysm

Stent optimization

Simulated annealing

\begin{abstract}
A B S T R A C T
A modern technique to treat cerebral aneurysms is to insert a flow diverter in the parent artery. In order to produce an optimal design of such devices, we consider a methodology combining simulated annealing optimization and lattice Boltzmann simulations. Our results surpass, in terms of stent efficiency, those obtained in the recent literature with an other optimization method. Although our approach is still in 2D, it demonstrates the potential of the method. We give some hint on how the 3D cases can be investigated.
\end{abstract}

(c) 2012 Elsevier B.V. All rights reserved.

\section{Introduction}

Cardiovascular disease, including heart disease and cerebral vascular disease, is one of the greatest causes of death in developed countries. Cerebral aneurysm is a vascular disorder that distends the vessel wall (see Fig. 1). In most cases, subarachnoid hemorrhage from a ruptured intracranial aneurysm impairs the quality of life of patients. In recent years endovascular treatments have attracted much attention due to their minimal invasiveness and good prognosis of patients.

The aim of endovascular treatments is to reduce the blood flow in an aneurysm by the placement of a device in the aneurysm (coil) and/or in the parent artery (stent or flow diverter). As a result of the flow reduction in the aneurysms, embolization by clot formation is expected to occur in the cavity, thus healing the aneurysm by its occlusion.

Aenis et al. reported that a stent has a capability of reduction of the flow in an aneurysm [1] and the reduction may lead to repair. Subsequent studies of sole stenting to reduce the flow more as a "flow diverter" rather than a "support for coiling" have been performed $[2,3]$. These studies have revealed that the effect of a stent on flow is not only dependent on porosity but also on the design of a stent.

\footnotetext{
* Corresponding author. Tel.: +4122379 0219.

E-mail address: Bastien.Chopard@unige.ch (B. Chopard).
}

In order to find a stent design that reduces the flow in aneurysm effectively, Srinivas et al. [4] applied an optimization procedure to determine the best stent structure across the neck of a 2D aneurysm, by sampling numerical simulations with different designs. This study shows that a better flow reduction is achieved when stent struts are concentrated at the edge of aneurysm neck rather than uniformly along the orifice.

Nakayama et al. investigated the size of strut with $80 \%$ porosity using a multi-objective genetic algorithm and kriging [5]. Finite volume method (FVM) was used and mesh generation turned out to be a delicate issue. Indeed all stent designs required handmade mesh generation, making it almost impossible to find out the best design with an automatic process. On the other hand, the Lattice Boltzmann (LB) method (see for instance [6]) uses a Cartesian mesh which can accommodate any stent design without having to generate the mesh manually. LB is a class of CFD methods for fluid simulations which represents a fluid as a set of fictitious particles moving and colliding on a regular lattice. It has been successfully used for many complex flow simulations. Hirabayashi et al. [7] applied the LB method to show the relationship between stent position and flow in aneurysm, thus illustrating the possibility of using LB simulation to study the effect of the stent fine scale details on blood circulation in cerebral aneurysms. In particular, when using stents with coarse struts, the flow reduction in aneurysm depends heavily on the stent position across the neck. Kim et al. [8] analyzed the shape of the strut cross section and concluded that rectangular struts reduce the flow more effectively than triangular or circular ones. 


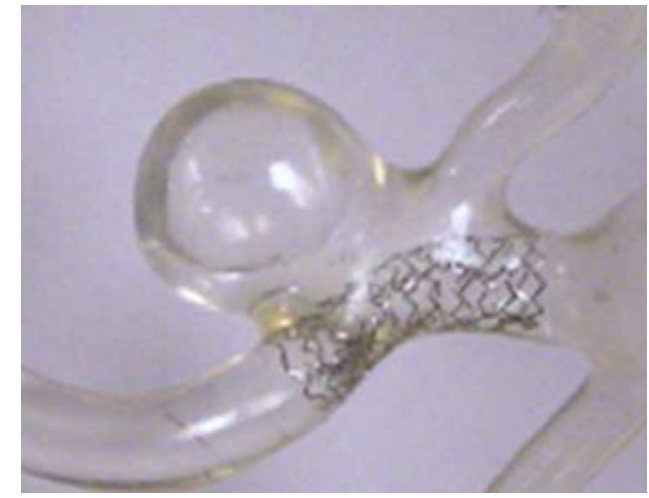

Fig. 1. An in vitro model of a cerebral aneurysm with a stent.

In this paper, we consider the possibility to use LB bloodflow simulations in combination with an optimization algorithm that can explore the design space of stents (or flow diverters), in a fully automated way. Optimization method may require a huge number of flow simulations to determine the best stent geometry. In the simulated annealing optimization that we consider here, simulations have to be done one after another. The advantages of the LB method in this respect is that it can be massively parallelized on huge scale computers, thus reducing significantly the wall clock time of each flow simulation.

Simulated annealing (SA) is a classical probabilistic metaheuristics applicable to hard optimization problems and especially to combinatorial optimization [9-11]. It can be shown to converge in probability to a global optimal, if the parameters are well chosen. In practice, SA searches effectively through a large solution set for good approximations of a global minimum. In our case, the objective function is defined as the average velocity norm inside the aneurysm. From one run to the next, the stent design is slightly modified, while preserving a prescribed porosity. If the new design produces a slower flow in the aneurysms cavity, the change is accepted. If not, the change is accepted with some probability, which depends on the current so-called "temperature", a parameter which guide the optimization process.

This paper focuses on a 2D ideal sidewall aneurysm in view of a feasibility study. In a forthcoming study, we shall report on the 3D, more realistic case.

\section{Method}

\subsection{Lattice Boltzmann simulations}

The Lattice Boltzmann (LB) method is an acknowledged alternative to standard computational fluid dynamics (CFD) techniques to solve the Navier-Stokes equations and other partial differential equations. It is described in several textbooks or review papers (see for instance $[6,12,13])$.

The LB method for hydrodynamics is a mesoscopic approach in which a fluid is described in terms of density distributions of idealized fluid particles moving and colliding on a regular lattice. These fluid particles can only take a finite number of possible velocities such that, in one time step of the dynamics, particles move from one lattice site to another.

Advantages of the LB method over more traditional numerical schemes are its simplicity, its flexibility to describe complex flows and its local nature (no need to solve a Poisson equation). LB solvers can be parallelized very naturally and scale well up to thousands of processors.

Here we perform 2D flow simulations using the standard D2Q9 lattice and the so-called single-time, BGK relaxation method $[6,12]$

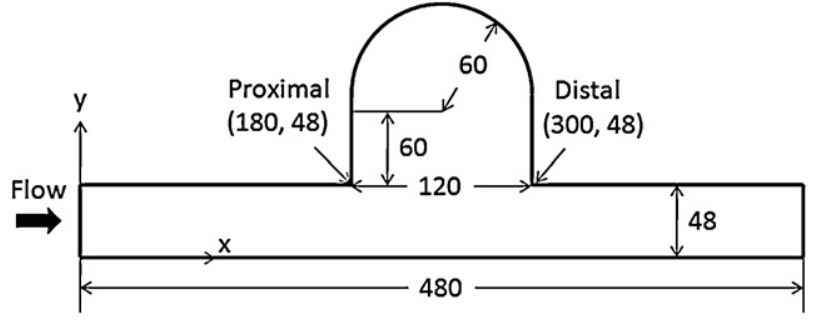

Fig. 2. Geometry of an ideal sidewall 2D aneurysm.

The problem we consider corresponds to the model of Srinivas et al. [4], illustrated in Fig. 2. The parent artery has length $L=40 \mathrm{~mm}$ and diameter $4 \mathrm{~mm}$. The aneurysm cavity has a depth and a neck of $10 \mathrm{~mm}$. The spatial discretization is chosen as $\Delta r=1 / 12 \mathrm{~mm}$, so that the full simulation fits in a lattice with $480 \times 168$ sites. The stent is modeled as a set of struts along the neck of the aneurysms. Each strut occupies one lattice site.

In order to impose no-slip boundary conditions on the walls of the vessel and the aneurysm, as well as on the lattice points that correspond to the stent struts, we use the well known bounce-back rule: the fluid particles bounce from were they came when hitting a no-slip lattice site (the velocity direction is reversed while the magnitude remains constant). The inlet boundary is defined as a velocity boundary condition, a steady parabolic profile with maximum velocity $U=0.15$, in lattice units. On the outlet, a pressure boundary is imposed. In order to achieve a Reynolds number of 300 , the kinetic viscosity is chosen as $v=U L / R e=0.024$, giving a relaxation time $\tau=(6 v+1) / 2=0.57$.

LB simulations are inherently time-dependent. The steady state is reached when the flow does not change anymore. Here, the steady state is assumed to be attained if the standard deviation of the average kinetic energy over the last 5000 time steps is less than $1.5 \times 10^{-6}$.

In this study, the open source software Palabos [14], version 0.7, which provides a flexible, highly parallelized and publicly available environment for the LB method, was used for the computational fluid dynamics (CFD) simulations. The optimization program developed in this study was run with 1, 15 and 20 CPUs.

\subsection{Optimization method}

The algorithm we used for stent shape optimization is the simulated annealing (SA) method. The steps of the algorithm are indicated in Fig. 3. In short, an initial stent geometry $S_{i n i t}$ is considered at the beginning of the process. The quality of this stent is evaluated through CFD simulations based on the LB method, by computing the average velocity $V$ in the aneurysm

$V=\frac{\sum_{\mathbf{r}} \sqrt{u_{x}^{2}(r)+u_{y}^{2}(t)}}{N}$

where the summation is carried out over all $N$ lattice sites $\mathbf{r}$ inside the aneurysm cavity.

The average velocity $V$ given by (1) is taken as the objective (or fitness) function of the problem. The goal is to find a stent design that minimizes it.

Note that other fitness functions can be considered. However, the property that an optimal stent should verify is still an open question. Velocity reduction in the cavity is a simple metric which can be used to demonstrate the potential of our approach and that allows for a comparison with the results found in [4].

As suggested in Fig. 3, the SA method proceeds by applying iteratively random modifications to the current stent geometry, until an optimal design is reached. The specificity of SA is to accept with some probability modifications that degrade the efficiency of the 


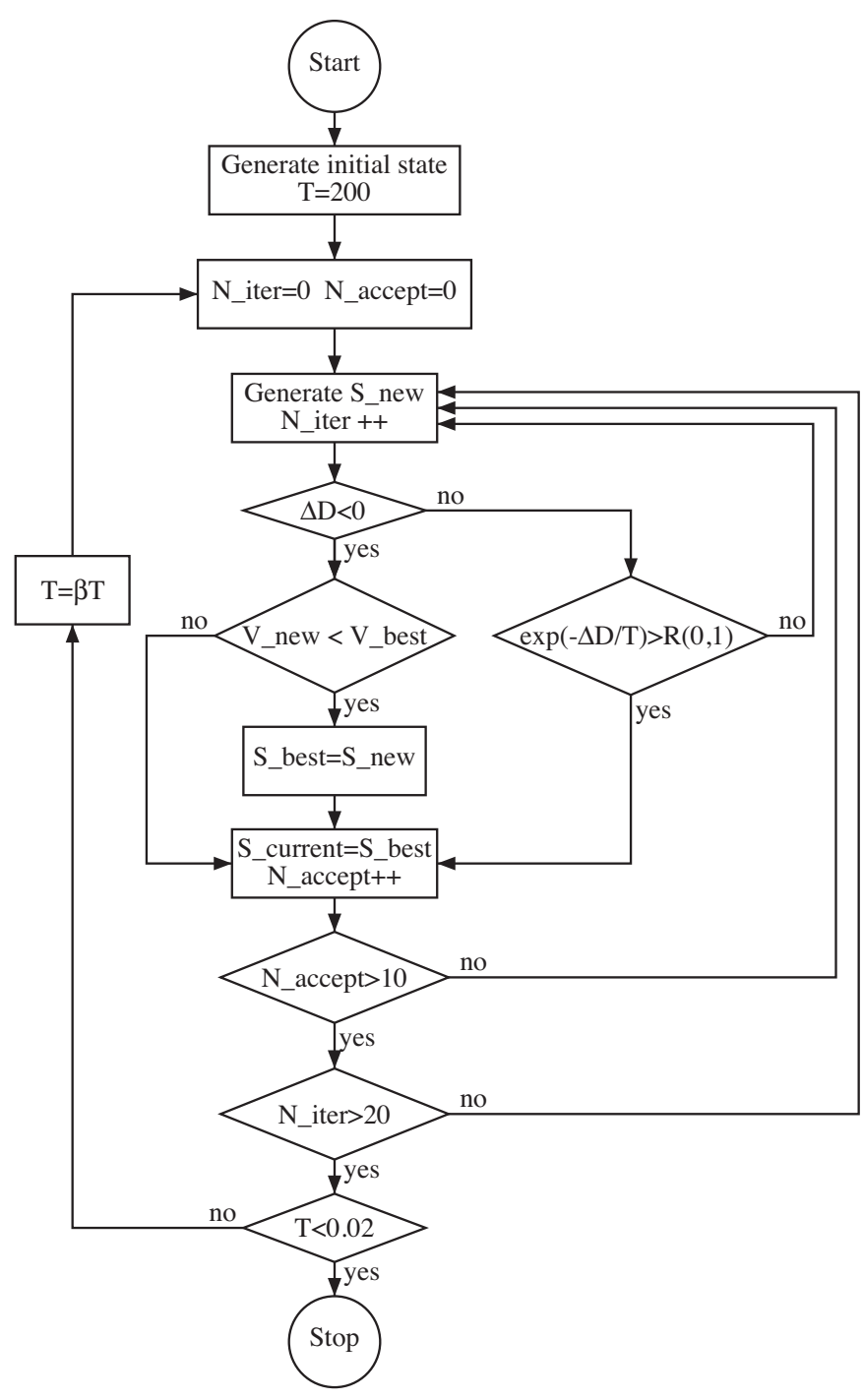

Fig. 3. Flow diagram of the simulated annealing optimization process, according to [15].

stent. Let us call $S_{\text {new }}$ a new stent geometry obtained from the current geometry $S_{\text {current }}$, through a small modification of its structure. In practice, this modification is obtained by exchanging randomly a lattice strut node with a fluid node, along the line defined by the aneurysm neck. In this way, the modification does not change the porosity of the stent.

The improvement of $S_{\text {new }}$ over $S_{\text {current }}$ is computed as the variation $\Delta D$ of the fitness function

$\Delta D=V_{\text {new }}-V_{\text {current }}$

The modification is accepted if

$\exp \left(\frac{-\Delta D}{T}\right)>R[0,1]$

where $R[0,1]$ is a random number between 0 and 1 and $T$ is the current "temperature" of the SA process. At the beginning, the temperature is high (here we start with $T=200$ ) and changes are often accepted even though they may degrade the quality of the stent. In this phase of the optimization, the exploration of the search space is dominant. However, when at a given temperature level $k$, no more improvement are observed on average, the temperature is decreased according to a prescribed temperature schedule. Here we chose $T_{k+1}=\beta T_{k}$ with $\beta=0.9$. The optimization process is stopped when $T=0.02$ is reached.

All along the optimization, the best geometry $S_{\text {best }}$ seen so far is remembered. Whenever $V_{\text {new }}<V_{\text {best }}, S_{\text {new }}$ becomes $S_{\text {best }}$. At the end of the process, $S_{\text {best }}$ is given as the optimal solution.

\section{Results}

In order to show the independence of the optimal stent on the initial condition of the SA search, four initial combinations for struts positions were considered, as depicted in Fig. 4. They are referred to as cases $A, B, C$ and $D$. In principle, if the optimization process is not blocked in a local minimum, all these initial configurations should eventually evolved towards the same global optimum. We chose a porosity of $80 \%$ (which is kept constant during the optimization procedure) to better compare our result with those reported in [4]. Note however that this porosity is higher than that of current flow diverters.

To express the results of our optimization in a dimensionless form, we use the flow reduction index $R_{f}$ defined as

$R_{f}=\frac{V_{w / o}-V_{\text {current }}}{V_{w / o}}$

where $V_{w / o}$ is the average velocity, computed from Eq. (1) without stent.

Fig. 5 shows the flow pattern without stent and with each of the four initial stents. The flow enters the aneurysm on the proximal side of the neck, and then goes out on the distal side. Secondary flow occurs inside the aneurysm. The initial stent $B$ leads to a fast inflow

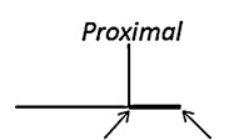

$(181,48) \quad(203,48)$

Case A

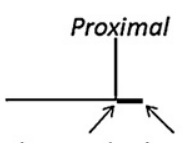

$(181,48) \quad(192,48)$

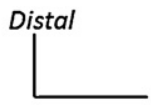

\section{A}

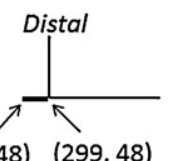

Case C

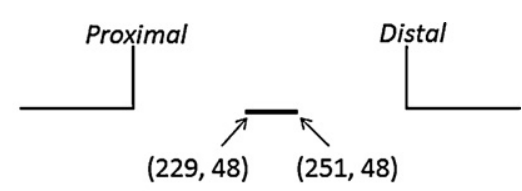

Case B

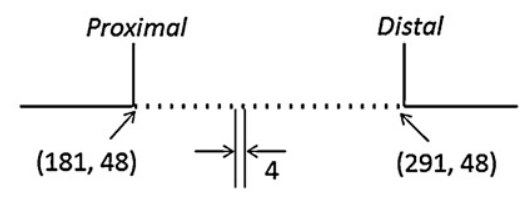

Case D

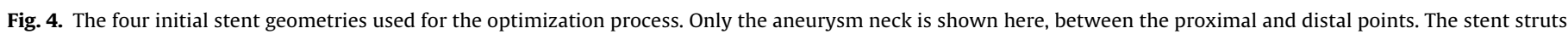
are indicated in bold. 


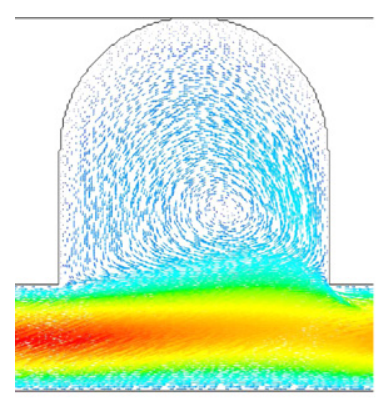

(without stent)

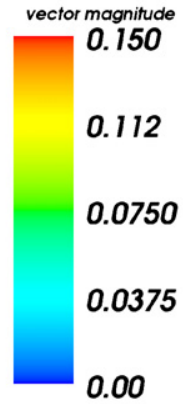

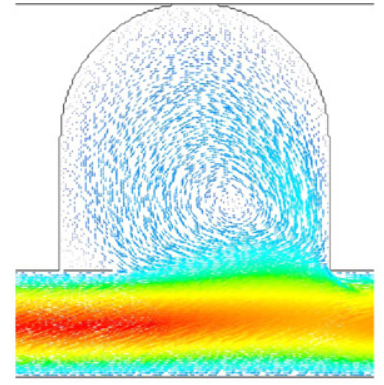

(Case A)

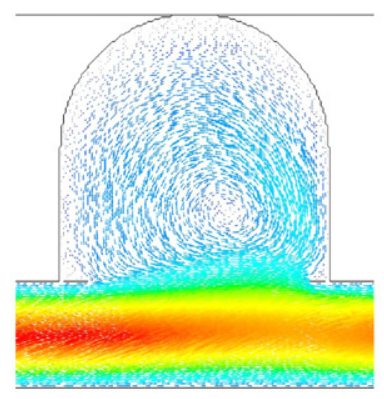

(Case C)

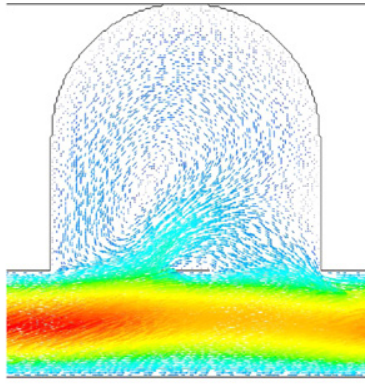

(Case B)

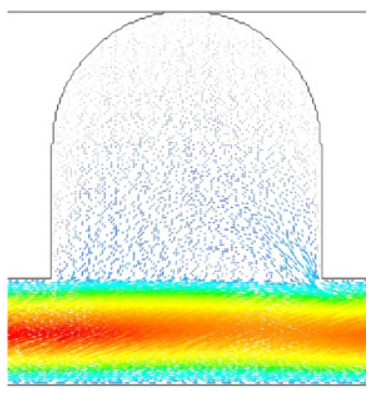

(Case D)

Fig. 5. Flow pattern before stent optimization. From left to right, top to bottom: situation without stent, then cases $A, B, C$ and $D$.

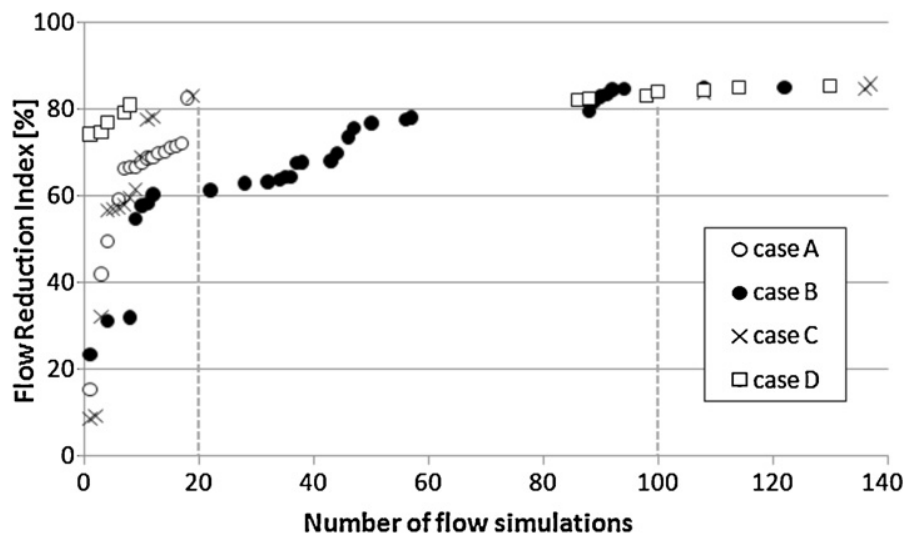

Fig. 6. Evolution of the flow reduction index as a function of the simulated annealing iteration, for the four initial stent geometries.

into the aneurysm. Initial stents $A$ and $C$ narrow the orifice of the aneurysm (see Fig. 4), thus reducing this inflow. Initial stent $D$ has a uniform distribution of struts and shows a good flow reduction of $R_{f}=74 \%$ even before the optimization process.
Fig. 6 shows the improvement of flow reduction on each stent shape during the optimization process. The horizontal axis represents the number of flow simulations, which counts both accepted and unaccepted stent modifications. The data points shown in this

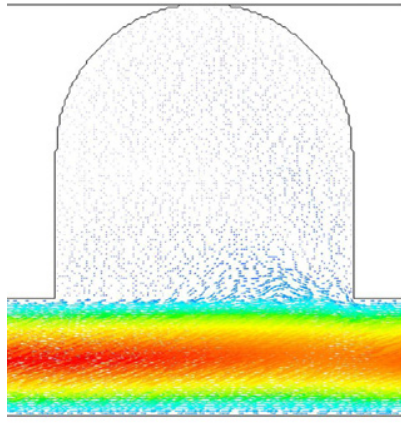

Case B
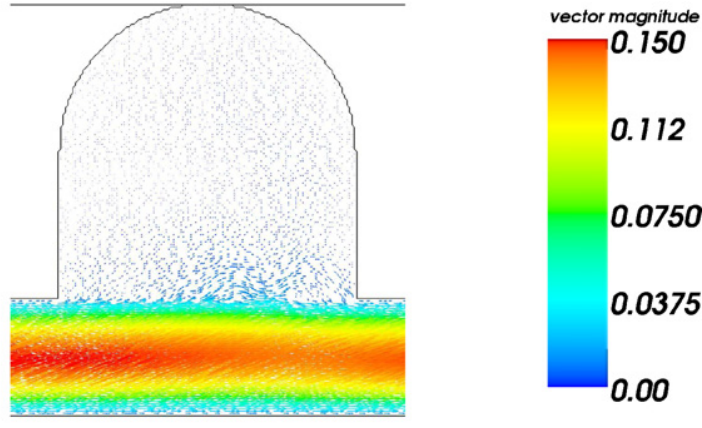

Case D

Fig. 7. Flow pattern (velocity vector) obtained with stent geometry $B$ (left) and $D$ (right), after optimization. 


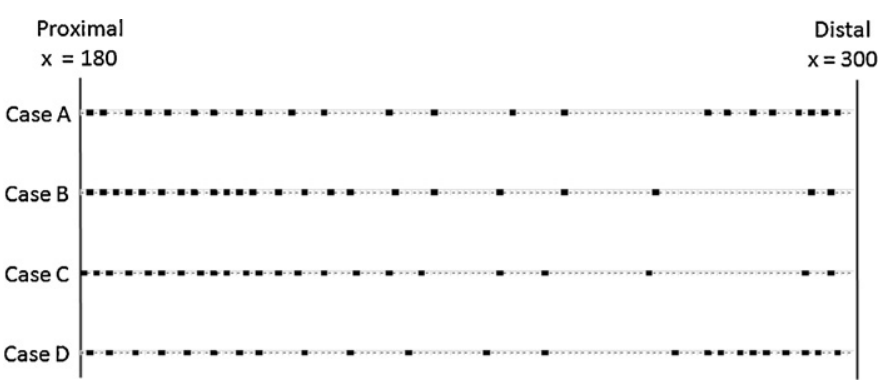

Fig. 8. Strut distribution along the aneurysm neck for the four initial stents, after the optimization process.

figure only correspond to iterations at which an improvement of $R_{f}$ has been accepted and $R_{f}$ has shown the best score. In this figure, the first 140 flow simulations are shown, however about 1800 of them are performed for each case. We observe a rapid improvement of the flow reduction within the first 100 flow simulations followed by a saturation phase. Stents $A, C$ and $D$ obtain $80 \%$ of flow reduction within 20 flow simulations. However, stent $B$ requires 100 simulations to reach $R_{f}>80 \%$.

After the execution of the optimization program, all stents evolve to the new geometry shown in Fig. 8, corresponding to the flow pattern illustrated in Fig. 7, for cases $B$ and $D$. In Fig. 8, black squares indicate the struts. Struts are distributed over the neck, with high density at both the proximal and distal ends. A low density region appears in the center. Flow reduction $R_{f}$ in the aneurysm reaches $89 \%$ in all cases. The highest reduction is obtained in case $B$ and the lowest one is obtained in case $D$. Table 1 summarizes the flow reduction $R_{f}$ observed before and after stent optimization for all cases.

The optimized stents $A, B, C$ and $D$ are not all exactly the same. This is likely due to premature convergence of the SA process, or to the existence of several optimal geometries. However, in terms of stent design, none of the design in Fig. 8 is satisfactory, due to the irregularity of strut distribution. A regularized stent can however be devised, in order to produce a consensus of the four geometries and enforce a more regular spacing of the struts.

The regularized stent can be thought of an average of the four optimized configuration. It is built as follows. From Fig. 8 one sees that all four stents have typically three regions with different average porosity: the proximal, central and distal ones. Fig. 9 shows the average, over the four stents, of the number of struts along the neck located in a sliding window containing 9 lattice sites (about $\ell=0.75$ $\mathrm{mm}$ ). The limits of the three regions are defined as the points where this number crosses the horizontal line at ordinate 2 , the value 2 being chosen arbitrarily. As illustrated in Fig. 10, the three regions obtained in this way are of size $39 \Delta r, 68 \Delta r$ and $12 \Delta r$, respectively. In addition, the average porosity in each of these regions are found to be $66.7 \%, 91.2 \%$ and $66.7 \%$. The porosity in these three regions can be achieved with 13, 6 and 4 struts, respectively. One can easily check that this choice produces an average porosity of $80 \%$ across the aneurysm neck, as required.

The reduction index $R_{f}$ for the regularized stent shown in Fig. 10 can then be obtained by a LB flow simulation. We find $R_{f}=88.6 \%$, which is less good than any of the optimal cases $A, B, C$ and $D$, but still a quite reasonable efficiency.

Table 1

Flow reduction $R_{f}$ for geometries $A, B, C$ and $D$, before and after optimization.

\begin{tabular}{lllcl}
\hline & Case $A[\%]$ & Case $B$ [\%] & Case C [\%] & Case $D[\%]$ \\
\hline Initial state & 15.3 & 23.3 & 8.5 & 74.3 \\
Optimized state & 89.4 & 89.8 & 89.5 & 89.2 \\
\hline
\end{tabular}

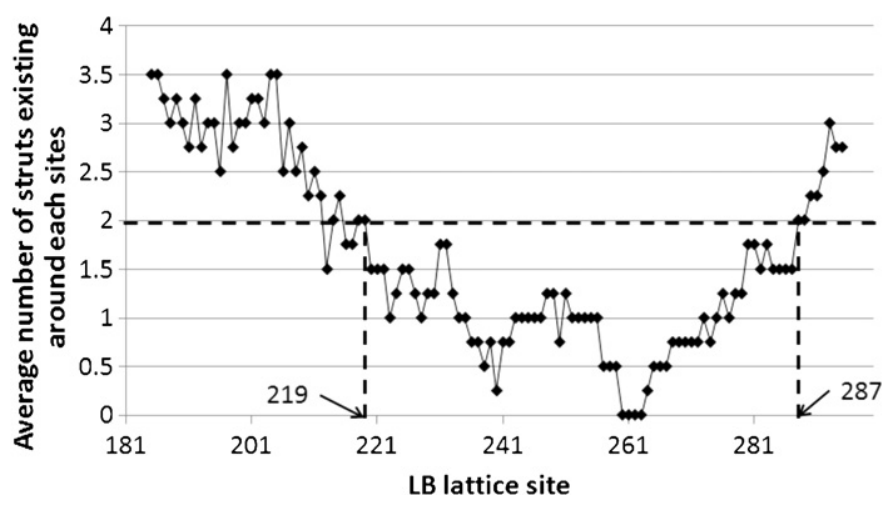

Fig. 9. Number of struts in an interval around each lattice node. The average is performed over the optimized geometries $A, B, C$ and $D$.

\section{Discussion}

This paper describes the combination of simulated annealing (SA) with lattice Boltzmann (LB) bloodflow calculations to discover the optimal stent design that best suit a given aneurysm geometry. Due to the simple Cartesian grid used in LB simulation, this combined approach is appropriate for a fully automated search process, as no manual fine tuning of the computational mesh around the stent struts is required each time a new stent design is tested.

LB simulations can be easily parallelized, and this is done automatically with the Palabos software. Therefore it is suited for large-scale parallel computation. In this study, for comparison purposes, the optimization program is performed for a $2 \mathrm{D}$ ideal aneurysm with both serial and parallel runs. The optimal stent design is obtained in $48 \mathrm{~h}$ with serial computing, whereas it takes 9 and $5 \mathrm{~h}$ with 10 and 20 CPUs, respectively. In principle, many more computing cores can be considered. Palabos has been shown to scale very well up to thousands of cores. Such a large scale parallelization will be needed when optimizing 3D geometries.

Theoretically, the SA method is able to find the global optimum if the initial temperature and cooling schedule are well chosen. Here we have considered a set of parameters that lead to a rather fast convergence but, very likely, a convergence to local optima. This is illustrated by the difference in the solution of the four initial stent geometries. However, the flow reduction index $R_{f}$, is quite similar between the four cases. It reaches $80 \%$ within the first 100 iterations and then stay more or less constant until the end of the program. The best stent we have obtained achieves $89 \%$ of velocity reduction.

The dependence of the above results on the mesh size is an important issue. In our case, we have checked that doubling the mesh resolution (i.e representing the struts by a $2 \times 2$ pixels instead of $1 \times 1$ ) modifies the velocity reduction obtained with the optimal stent by less than $1 \%$.

It is interesting to compare this result with another optimization approach using the same aneurysm. Srinivas et al. [4] obtained about $76 \%$ of velocity reduction with an optimized stent, whose porosity is $80 \%$ and consisting of five wide struts, instead of the 23 thin ones, we obtain here. The CPU time reported by Srinivas et al. is from $2 \mathrm{~min}$ to $60 \mathrm{~min}$ for one geometry, depending upon the case considered and the type of computer used. The method requires

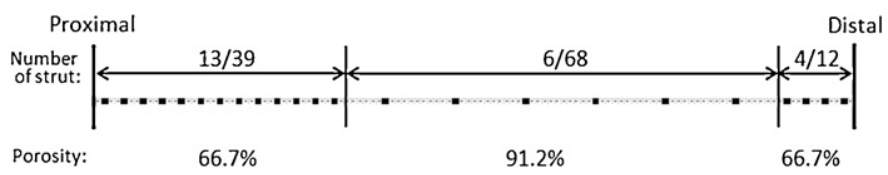

Fig. 10. The regularized stent, obtained as a regular consensus of optimal stents $A$, $B, C$ and $D$. 


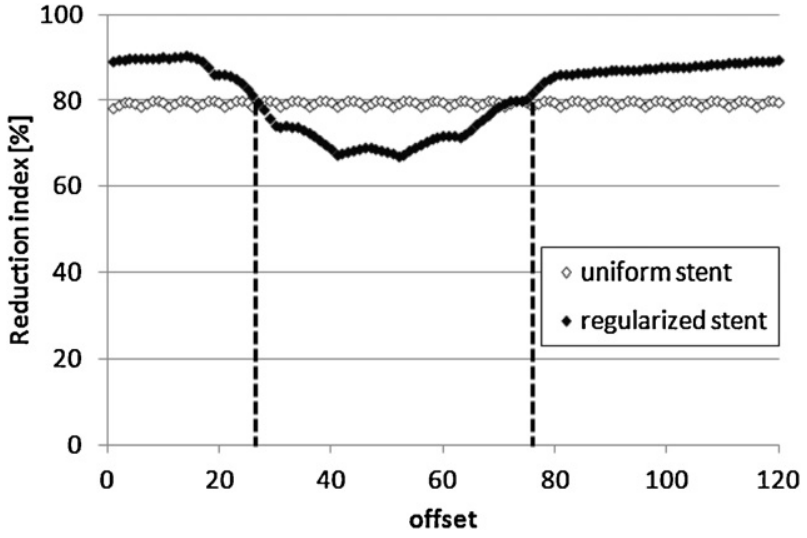

Fig. 11. Variation of the flow reduction obtained with the optimal regularized stent as a function of its positioning along the neck. This variation is compared to the flow reduction achieved with a uniform stent with the same porosity.

the calculation of the flows patterns for 60 different geometries. Mesh and geometry are constructed by an operator and the operation time should be considered. On a personal computer, one can thus assume that the CPU time is around $60 \times 60 \mathrm{~min}$, i.e. $60 \mathrm{~h}$. This more than the CPU time of our approach on a personal computer $(48 \mathrm{~h})$, and it produces a less optimal stent than ours.

All of the optimized stents we have generated have a nonuniform distribution of struts. They are dense at both proximal and distal ends, with a sparse region in the middle. The dense part at the proximal end is longer than the distal one. This feature is consistent with the inflow and outflow patterns observed in aneurysm geometry such as the one we consider here. Anzai et al. [16] have reported that struts placed where the flow enters the aneurysm decrease the flow more effectively than struts placed where the flow leaves the cavity. This suggests that the optimized stent will become dense at the inflow zone even in realistic 3D geometry.

A non-uniform stent raises the question of the sensitivity of the flow reduction index on the stent position along the neck. As shown in Fig. 11, the regularized stent provides a higher $R_{f}$ than a uniform one within a tolerance of two to three millimeters from its optimal position. However, when the high porosity part of the regularized stent is situated at proximal end of the neck, $R_{f}$ becomes lower than with an uniform stent with $80 \%$ of porosity.

Fig. 6 shows the variation of the number of SA iterations needed to converge to the optimal state, from different initial conditions. This variation suggests that starting with an initial stent geometry close to the optimal one will significantly save computational time. This might be important for 3D optimizations.

In this study, the flow is steady and Newtonian. This can be easily changed by adapting the boundary conditions of the flow solver and to turn on a non-Newtonian rheology in the LB solver (see for instance $[17,18])$. Of course, time-dependent flow simulations are expected to be more costly than steady ones. However, since the LB method is inherently time-dependent, this will not add so much extra computational time to the full optimization process. In the near future, we plan simulations with pulsatile flows to determine how the optimal stent structure changes with respect to the steady case.

Of course, the present approach should be extended to 3D to be of practical importance. There are several issues to be solved in relation to the vast increase of the search space and computational load. The 3D extension is part of an ongoing study, the European FP7 VPH project THROMBUS. In addition to computational time, the challenge of the 3D case is the stent representation in the computer and the definition of the elementary changes required by the SA algorithm.
We shall first consider the ideal side-wall aneurysm, then realistic aneurysm obtained from medical imaging with the purpose to achieve tailor-made medicine.

Several approaches can be considered to address the 3D problem. We can use the same method as in the 2D case and interpret the resulting spatial distribution of struts as an indication of the zones with optimal porosity. Or we can consider only geometry modifications that guarantee the continuity of the wires making up the stent. Finally, if the stent geometry can be described by a mathematical parametrization, the SA can be used to find the optimal values of these parameters.

Note finally that a proper choice of objective function is required to make meaningful predictions on stent performance. However, it is still unknown which quantities are critical for the growth and the rupture of an aneurysm, or are needed to induce thrombosis in the cavity. Thus, at the present time, we considered the singleobjective optimization specified by the minimization of the average velocity inside the aneurysm. But our approach can be equally well applied with any objective function, such as the oscillatory shear index (OSI) [19], flow complexity [20], gradient oscillatory number (GON) [21], and even multi-objective problems.

\section{Conclusions}

Stent optimization is a step toward an era of tailor-made medicine for each individual.

In this paper, stent design based on the combination of Lattice Boltzmann flow simulations and Simulated Annealing optimization was developed for a 2D ideal aneurysm geometry. Although only 3D stent optimization is relevant for medical applications, the 2D case presented here serves as a feasibility study. Our results on the full 3D problem are still in an early stage and will be reported in the future.

Our results can be summarized as follows. After execution of the optimization process with four initial stent geometries, all of them were reshaped through a succession of elementary modification that, eventually, produce over $89 \%$ of flow reduction inside the aneurysm cavity. As a next step, we proposed a regularized stent geometries which can be thought of as a design average of the four optimized stents. This consensus stent is made of three sections, alternating dense and sparse porosity. This stent produces $88.6 \%$ of flow reduction, in good agreement with the reduction reached by the four optimal stents.

Except from initial stent condition $B$, a flow reduction of $80 \%$ is obtained within the first 20 iterations of the simulated annealing process. Case $B$ required over 80 modifications until the flow reduction reaches that level. This difference of effort to obtain a good stent efficiency suggests the importance of choosing an appropriate initial state.

The present approach can be fully automated and run on large scale parallel computers. It achieves a significantly better stent design than the one obtained in a recent study by Srinivas et al. [4], with less CPU requirements on a serial machine.

\section{Acknowledgements}

This study was supported by a Research Fellowship of the Japan Society for the Promotion of Science for Young Scientists. Part of the work was carried out under the Collaborative Research Project of the Institute of Fluid Science, Tohoku University. We thank David Cervini for his study of the convergence of our results on the mesh resolution. This work is part of the THROMBUS European FP7-ICT project no. 269966. 


\section{References}

[1] M. Aenis, et al., Modeling of flow in a straight stented and nonstented side wall aneurysm model, J. Biomech. Eng.-Trans. ASME 119 (2) (1997) 206-212.

[2] S. Appanaboyina, et al., Simulation of intracranial aneurysm stenting: Techniques and challenges, Comput. Methods Appl. Mech. Eng. 198 (45-46) (2009) 3567-3582.

[3] L. Augsburger, et al., Effect of flow diverter porosity on intraaneurysmal blood flow, Clin. Neuroradiol.[[nl]]Klinische Neuroradiologie 19 (3) (2009) 204-214.

[4] K. Srinivas, et al., Two-dimensional optimization of a stent for an aneurysm, J. Med. Dev. Trans. ASME 4 (2) (2010) 021003.

[5] T. Nakayama, et al., Development of stent strut pattern for cerebral aneurysm, in: Proceedings of the 3rd ASME 2010 Joint US-European Fluids Engineering Summer Meeting and 8th International Conference on Nanochannels, Microchannels, and Minichannels:FEDSM/ICNMM, 2010, p. 30592.

[6] S. Succi, The Lattice Boltzmann Equation, For Fluid Dynamics and Beyond, Oxford University Press, 2001.

[7] M. Hirabayashi, M. Ohta, D.A. Rufenacht, B. Chopard, Characterization of flow reduction in an aneurysm due to a porous stent, Phys. Rev. E 68 (2003) 021918.

[8] Y. Kim, X. Xu, J. Lee, The effect of stent porosity and strut shape on saccular aneurysm and its numerical analysis with lattice Boltzmann method, A. Biomed. Eng. 38 (7) (2010) 2274-2292.

[9] S. Kirkpatrick, Optimization by simulated annealing-quantitative studies, J. Stat. Phys. 34 (1984) 5-6.

[10] D. Johnson, et al., Optimization by simulated annealing-an experimental evaluation, Oper. Res. (1991).

[11] D. Bertsimas, J. Tsitsiklis, Simulated annealing, Stat. Sci. 8 (1) (1993) 10-15.

[12] B. Chopard, P. Luthi, A. Masselot, A. Dupuis, Cellular automata and lattice Boltzmann techniques: an approach to model and simulate complex systems, Adv. Complex Syst. 5 (2) (2002) 103-246, http://cui.unige.ch/ chopard/ FTP/CA/acs.pdf.

[13] C. Aidun, J. Clausen, Lattice-Boltzmann method for complex flows, Ann. Rev. Fluid Mech. 42 (1) (2010) 439-472.

[14] http://http://www.palabos.org/.

[15] P.J.M. Laarhoven, E.H.L. Aarts (Eds.), Simulated annealing: theory and applications, Kluwer Academic Publishers, Norwell, MA, USA, 1987.

[16] $\mathrm{H}$. Anzai, et al., The effect of $3 \mathrm{~d}$ visualization on optimal design of strut position of intracranial stent, in: Proceedings of the 3rd ASME 2010 Joint US-European Fluids Engineering Summer Meeting and 8th International Conference on Nanochannels, Microchannels, and Minichannels:FEDSM/ICNMM, 2010, p. 30591.

[17] B. Chopard, R. Ouared, D.A. Ruefenacht, H. Yilmaz, Lattice Boltzmann modeling of thrombosis in giant aneurysms, Int. J. Mod. Phys. C 18 (2007) 712-721.

[18] O. Malaspinas, G. Courbebaisse, M. Deville, Simulation of generalized newtonian fluids with the lattice Boltzmann method, Int. J. Mod. Phys. C 18 (2007) 1939.

[19] D. Ku, D. Giddens, C. Zarins, S. Glagov, Pulsatile flow and atherosclerosis in the human carotid bifurcation-positive correlation between plaque location and low and oscillating shear-stress, Arteriosclerosis 5 (3) (1985) 293-302.

[20] J.R. Cebral, F. Mut, J. Weir, C.M. Putman, Association of hemodynamic characteristics and cerebral aneurysm rupture, Am. J. Neuroradiol. 32 (2) (2011) 264-270.

[21] Y. Shimogonya, T. Ishikawa, Y. Imai, N. Matsuki, T. Yamaguchi, Can temporal fluctuation in spatial wall shear stress gradient initiate a cerebral aneurysm? A proposed novel hemodynamic index the gradient oscillatory number (GON), J. Biomech. 42 (4) (2009) 550-554.

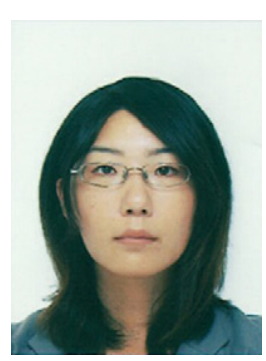

Hitomi Anzai is a PhD student, Graduate School of Engineering. Tohoku University. She has graduated in School of Engineering, Tohoku University (2005-2009), and then entered Graduate School of Engineering, Tohoku University (2009-present). She is a research fellow of the Japan Society for the Promotion of Science for Young Scientists. Her research field is computed fluid dynamics on/in intracranial aneurysm.

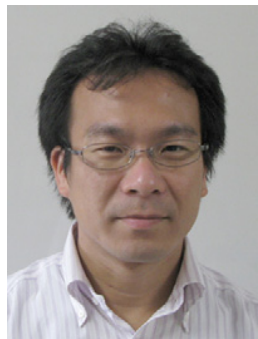

Makoto Ohta received a PhD in Biopolymers from Department of Polymer Chemistry, Kyoto University (2001). He then worked in Institute for Frontier Medical Sciences, Kyoto University (2001) and spent several years at the University Hospital of Geneva as a research fellow and post-doctoral position (2001-2004). He is now associate professor at Institute of Fluid Science, Tohoku University (2005-present). His research topics are design development of intracranial stent based on blood flow dynamics, and development of biomodelling for evaluation of medical devices. He published over 50 publication based on the biomechanics and biomaterial fields.

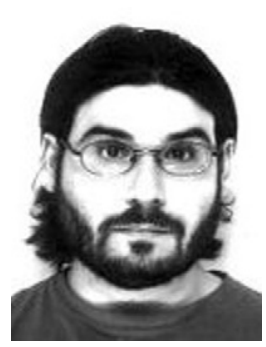

Dr. Jean-Luc Falcone is a researcher in the University of Geneva. He is a biologist with a strong background in computational science and data-mining methods for life sciences. Since June 2010, he has been working for the lemanic center CADMOS as High Performance Computing analyst.

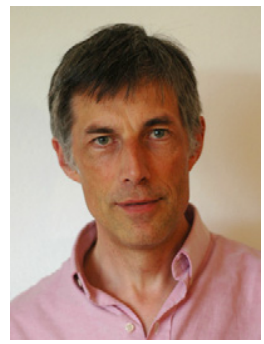

Bastien Chopard received a $\mathrm{PhD}$ in theoretical physics from the University of Geneva (1988). He then spent two years in the Laboratory for Computer Science, at the Massachusetts Institute of Technology and one year at the Center for High Performance Computing in the Research Center in Jülich, Germany. He is now professor at the Department of Computer Sciences of the University of Geneva. His research interests concern the modeling and simulation of complex systems on parallel computers. A large part of his work concerns the field of cellular automata, lattice gas and lattice Boltzmann techniques. Numerical simulation of biomedical applications is an important part of his current research activities. He published about 200 papers and is co-author of a textbook on Cellular Automata modeling of Physical systems (Cambridge University Press, 1998). 